\title{
Erratum to: Significance of the urokinase- type plasminogen activator and its receptor in the progression of focal segmental glomerulosclerosis in clinical and mouse models
}

\author{
Jin-Shuen Chen ${ }^{1}$, Li-Chien Chang ${ }^{2}$, Chung-Ze Wu ${ }^{3}$, Tzu-Ling Tseng ${ }^{4}$, Jui-An Lin ${ }^{5}$, Yuh-Feng Lin ${ }^{3,5}$ \\ and Chao-Wen Cheng ${ }^{5,6^{*}}$
}

\section{Erratum}

Following publication of this article [1] it has come to our attention that one of the supported grants from the Tri-Service General Hospital was omitted:

"We thank all subjects who participated in the study. This work was supported by grants from the National Defense Medical Center, the Tri-Service General Hospital (TSGH-C103-007-S02), and the Ministry of Science and Technology of Taiwan (MOST 102-2314-B-016-012-), Taiwan. This work was also partly supported by a grant from Taipei Medical University (TMU100-AE3-Y06). The authors thank Ms. Hsin-Yi Tsai for her technical support".

This should have been presented as: "We thank all subjects who participated in the study. This work was supported by grants from the National Defense Medical Center, the Tri-Service General Hospital (TSGH-C103007-S02, TSGH-C104-007-007-S02), and the Ministry of Science and Technology of Taiwan (MOST 1022314-B-016-012-), Taiwan. This work was also partly supported by a grant from Taipei Medical University (TMU100-AE3-Y06). The authors thank Ms. Hsin-Yi Tsai for her technical support".

The authors would like to sincerely apologize for any inconvenience or confusion this may have caused.

\begin{abstract}
Author details
'Division of Nephrology, Department of Internal Medicine, Tri-Service General Hospital, National Defense Medical Center, No. 325, Section 2, Chenggong Road, Neihu District, Taipei 114, Taiwan. ${ }^{2}$ School of Pharmacy, National Defense Medical Center, Taipei, Taiwan. ${ }^{3}$ Department of Internal Medicine, Shuang Ho Hospital, Taipei Medical University, New Taipei City, Taiwan. ${ }^{4}$ Biomedical Technology \& Device Research Laboratories, Industrial Technology Research Institute, Hsinchu, Taiwan. ${ }^{5}$ Graduate Institute of Clinical Medicine, College of Medicine, Taipei Medical University, No. 250, Wu-Xing Street, Taipei 110, Taiwan. ${ }^{6}$ Graduate Institute of Medical Sciences, National Defense Medical Center, Taipei, Taiwan.
\end{abstract}

Received: 29 June 2017 Accepted: 29 June 2017

Published online: 19 July 2017

\section{Reference}

1. Chen J-S, Chang L-C, Wu C-Z, Tseng T-L, Lin J-A, Lin Y-F, Cheng C-W. Significance of the urokinase-type plasminogen activator and its receptor in the progression of focal segmental glomerulosclerosis in clinical and mouse models. J Biomed Sci. 2016;23:24.

* Correspondence: ccheng@tmu.edu.tw

${ }^{5}$ Graduate Institute of Clinical Medicine, College of Medicine, Taipei Medical University, No. 250, Wu-Xing Street, Taipei 110, Taiwan

${ }^{6}$ Graduate Institute of Medical Sciences, National Defense Medical Center

Taipei, Taiwan 